\title{
Automatic Computing Methods for Special Functions. Part II. The Exponential Integral $E_{n}(x)$
}

\author{
Irene A. Stegun and Ruth Zucker \\ Institute for Basic Standards, National Bureau of Standards, Washington, D.C. 20234
}

(September 4, 1974)

\begin{abstract}
Accurate, automatic, efficient methods for computing the exponential integral $E_{n}(x)$ are detailed and implemented in an American National Standard FORTRAN program. The driver program and test results are also included.
\end{abstract}

Key words: Computer programs; continued fraction; exponential integral; key values; recurrence relation.

\section{Introduction}

The exponential integral itself occurs in many physical problems and often many other integrals are expressible in terms of the exponential integral. In view of its importance and the difficulties accompanying the repeated application of its recurrence relation, we have chosen this integral as Part II. (For Part I, see J. of Research NBS, Vol. 74B, July-September 1970, pp. 211-224.)

Accurate, efficient, automatic computing methods implemented in American National Standard FORTRAN, covering the entire range of machine legitimate arguments and/or functional values will be supplied. The number of terms in series, the number of convergents in an iterative process, etc., are all determined by the computer as a function of word length, arguments, the accuracy desired, etc. In cases of error returns, more realistic results will be returned. To further ensure correct limiting values of related functions, the proper analytic behavior of the function will always be retained. The driver program and test results are also included.

\section{Mathematical Formulas}

Relevant formulas are collected here for completeness and ease of reference. In keeping with the convention of the Handbook [1], ${ }^{1} x$ here is a real variable.

A. Definition

$$
\begin{aligned}
E_{n}(x) & =\int_{1}^{\infty} t^{-n} e^{-x t} d t \quad(n=0,1,2, \ldots ; x>0) \\
& =\int_{0}^{1} u^{n-2} e^{-x / u} d u \\
& =x^{n-1} \int_{x}^{\infty} t^{-n} e^{-t} d t .
\end{aligned}
$$

AMS Subject classification: 33-04, 65D20.

${ }^{1}$ Figures in brackets indicate references on page 205. 


\section{B. Series Expansion}

$$
\begin{gathered}
E_{n}(x)=\frac{(-x)^{n-1}}{(n-1) !}[-\ln x+\Psi(n)]-\sum_{\substack{m=0 \\
m \neq n-1}}^{\infty} \frac{(-x)^{m}}{m !(m-n+1)} \quad(x>0) \\
\Psi(1)=-\gamma, \Psi(n)=-\gamma+\sum_{m=1}^{n-1} \frac{1}{m} \quad(n>1)
\end{gathered}
$$

$\gamma($ Euler's constant $)=.5772156649 \ldots$.

$$
\begin{aligned}
& \text { C. Continued Fraction } \\
& E_{n}(x)=e^{-x}\left(\frac{1}{x+1} \frac{n}{1+} \frac{1}{x+} \frac{n+1}{1+} \frac{2}{x+} \ldots\right) \quad(x>0) . \\
& \text { D. Asymptotic Expansion } \\
& E_{n}(x) \sim \frac{e^{-x}}{x}\left[1-\frac{n}{x}+\frac{n(n+1)}{x^{2}}-\frac{n(n+1)(n+2)}{x^{3}}+\ldots\right] . \\
& \text { E. Special Values } \\
& E_{n}(0)=\frac{1}{n-1} \quad(n>1) \\
& E_{0}(x)=\frac{e^{-x}}{x} .
\end{aligned}
$$

F. Recurrence Relation

$$
E_{n+1}(x)=\frac{e^{-x}}{n}-\frac{x}{n} E_{n}(x) \quad n=1,2,3, \ldots
$$

G. Differentiation Formula

$$
\frac{d^{k}}{d x^{k}} E_{n}(x)=(-1)^{k} E_{n-k}(x) \quad n=1,2,3, \ldots
$$

H. Inequalities

$$
\begin{gathered}
(x>0, n=1,2,3, \ldots) \\
\frac{n-1}{n} E_{n}(x)<E_{n+1}(x)<E_{n}(x) \\
\frac{1}{x+n}<e^{x} E_{n}(x) \leqslant \frac{1}{x+n-1} .
\end{gathered}
$$




\section{Related Functions}

Incomplete Gamma Function

$\Gamma(1-n, x)=x^{1-n} E_{n}(x)$

Confluent Hypergeometric Function

$U(n, n, x)=e^{x} x^{1-n} E_{n}(x)$.

\section{Method}

Examination of the recurrence relation indicates that an independent computation must be carried out for at least one value of $n \geqslant 1$. The function $E_{n}(x)$ is always positive, for real $x$ has no zeros, and is a slowly decreasing function with increasing $n$ and/or $x$. Repeated application of the recurrence relation therefore will yield an increasing round off error even if, with more complicated scaling, both forward and backward recurrence relations are used starting at $n=[x]$. Generally, in physical applications the first few orders only are needed. Consequently, we have chosen an independent computing method valid for all orders.

The implementing American National Standard FORTRAN program has been set up in such a way as to require a minimal number of changes for varying precision, either single or double precision on the same or different computers. The program checks for positive arguments $n(=R N)$ and $x$ and integer $n$. If either is negative, an error indicator is set $($ IERR $=1)$ and an impossible value, the negative of the maximum machine value $(=\mathrm{RINF})$, is returned for both functional values, $E_{n}(x)(=\mathrm{ENX})$ and $e^{x} E_{n}(x)(=\mathrm{EXPENX})$. If $n>\mathrm{RMAXI}$, the maximum integer convertible to a floating point number, $n$ is assumed to be an integer. If $n \leqslant$ RMAXI, an integer test is applied to $R N$. If it fails the test, IERR is set equal to 2, and RINF is returned for both functional values. To assure ready portability, additional tests are performed for negative zero and allowance for round off errors due either to machine arithmetic or the system being used.

The special cases are treated independently. When $n=0$, to avoid machine difficulty, $x$ is tested against the reciprocal of the maximum machine value; if $x$ equals, or is less than that number, the maximum value is returned for both functional values. If $x$ is greater, then $\mathrm{ENX}=e^{-x} / x$ and $\operatorname{EXPENX}=1 / x$. When $x=0$, and $n=0$ or $1, \mathrm{ENX}=\mathrm{EXPENX}=\mathrm{RINF}$; for $n>1, \mathrm{ENX}=\mathrm{EXPENX}$ $=1 /(n-1)$.

Computation with the series results in a greater round-off error in the neighborhood of $x=1$, in particular for small values of $n$. Even if the form of the series were changed from the present alternating form, there is still a loss due to the logarithmic term. While the continued fraction is convergent for $x>0$, the number of terms increases rapidly as $x$ approaches 0 . The integrand is not particularly amenable to automatic numerical integration by any of the low order formulas. In general $(n \leqslant$ RMAXI) the most accurate and efficient methods of automatic computation are the alternating power series for $x \leqslant 1$ (ULPS) and the "even" form of the continued fraction for $x>1$.

For $1 \leqslant n \leqslant$ RMAXI and $0<x \leqslant 1$, the power series is used in the following form

$$
E_{n}(x)=\mathrm{ENX}=\sum_{M=0}^{K} T_{M}=\mathrm{SUM}+T M(M=0,1, \ldots, K)
$$

where $T_{M}=\frac{-(-x)^{M}}{M !(M-n+1=D)}=\frac{\text { PTERM }}{D} \quad$ for $M \neq n-1$

and $T_{M}=\operatorname{PTERM}[\operatorname{LOG}(x)-\operatorname{PSI}(n)]$ for $M=n-1$.

PTERM is obtained by recurrence with $\operatorname{PTERM}(0)=-1$ and $\operatorname{PTERM}(M+1)=$ PTERM $(M) \cdot(-x) / M+1$. 
Rather than compute a previously determined fixed number of terms, it is valid, provided $M \neq n-1$, to terminate the series when the relative error, computed as $|T M / \mathrm{SUM}|$ is less than or equal to a prescribed tolerance (=TOLER). In the present program, TOLER $=2^{-N B M}$, where NBM is the maximum number of binary digits in the mantissa of a floating point number. It may, however, be set to the accuracy desired.

Since the series is an alternating one, SUM may equal zero at some $M$. However, since the function has no zeros for real $x$, the relative error test may be safely bypassed if SUM $=0$ and additional terms computed. In our manner of evaluating the series, $T M$ may likewise be zero if $\log (x)=\Psi(n)$. (Values of $e^{\Psi(n)}$ for $n=1(1) 10$ are given in sec. 10.) The logarithmic term enters the computation for $n \leqslant 2$ since at least two terms of the series must be used with the above relative error test. Consequently, using the power series for $x \leqslant 1$, this situation will not then be critical when $n=1$ and $x \approx 0.56$. However, since the code may be used for experimental purposes, the relative error test is not applied to the logarithmic term.

For $n \geqslant 1$ and $x>1$, and $n>$ RMAXI, $x>0$, the continued fraction in its "even" form

$$
\begin{aligned}
E_{n}(x) & =e^{-x}\left[\frac{1}{x+n-} \frac{1 \cdot n}{x+n+2-} \frac{2(n+1)}{x+n+4-} \ldots\right] \\
& =e^{-x} F=e^{-x} \operatorname{EXPENX}
\end{aligned}
$$

is evaluated in the forward direction. The first convergent $F_{1} / G_{1}=A_{1} / B_{1}$ where $A_{1}=1, A_{M}=$ $-(M-1)(n+M-2), B_{M}=x+n+2(M-1)$. If we define

$$
F_{-1}=1, F_{0}=0, G_{-1}=0 \text { and } G_{0}=1
$$

then successive convergents $F_{M} / G_{M}$ for $M=1,2, \ldots$ may be obtained by the following recurrence relation

$$
\begin{aligned}
& F_{M}=B_{M} F_{M-1}+A_{M} F_{M-2} \\
& G_{M}=B_{M} G_{M-1}+A_{M} G_{M-2} .
\end{aligned}
$$

Since in the basic continued fraction $A_{M}$ and $B_{M}$ are always positive with $A_{M} \geqslant 1, F_{M}$ and $G_{M}$ will always be positive and increasing with increasing $M$. Care must then be taken to ensure that overflow does not occur in generating the successive convergents. For the "even" form of the continued fraction, since $A_{M}<0$ for $M>1$, it is sufficient to check that $G_{M}$ is always less than $\mathrm{RINF} / B_{M}$. If $G_{M}$ is greater, then $F_{M}, F_{M-1}, G_{M}$ and $G_{M-1}$ are all scaled by dividing by $B_{M}$.

Since, for the above continued fraction, the successive convergents form a monotonically increasing sequence, if through round-off errors,

$$
1-\frac{\left(F_{M-1} / G_{M-1}\right)(=\mathrm{PREV})}{F_{M} / G_{M}} \leqslant 0 \quad\left(F_{M} / G_{M} \leqslant F_{M-1} / G_{M-1}\right)
$$

then $F_{M-1} / G_{M-1}=F$.

If $0<1-\frac{F_{M-1} / G_{M-1}}{F_{M} / G_{M}} \leqslant$ TOLER $\left(=2^{-N B M}\right)$, then $F_{M} / G_{M}=F$.

The following table gives an indication of the number of terms needed to obtain maximum machine accuracy for particular values of NBM, $x$ and $n$ with the various methods of computation. 


\begin{tabular}{|c|c|c|c|c|}
\hline \multirow{3}{*}{$\begin{array}{l}\text { Method } \\
x=1\end{array}$} & \multicolumn{4}{|c|}{ Number of Terms } \\
\hline & \multicolumn{2}{|c|}{$\mathrm{NBM}=27$} & \multicolumn{2}{|c|}{$\mathrm{NBM}=60$} \\
\hline & $n=1$ & $n=10$ & $n=1$ & $n=10$ \\
\hline Power Series & 13 & 14 & 21 & 22 \\
\hline Continued Fraction & 26 & 17 & 113 & 76 \\
\hline $\begin{array}{l}\text { Numerical Integration } \\
\text { (Trapezoidal or Simpson's } \\
\text { Rule) }\end{array}$ & 129 & 513 & 16385 & 65537 \\
\hline$x=22$ & & & & \\
\hline Asymptotic Expansion & 22 & & & \\
\hline Continued Fraction & 5 & 6 & 12 & 16 \\
\hline $\begin{array}{l}\text { Numerical Integration } \\
\qquad x=44\end{array}$ & 2049 & & & \\
\hline Asymptotic Expansion & 10 & 35 & 45 & ... \\
\hline Continued Fraction & 4 & 5 & 9 & 11 \\
\hline$x=70$ & & & & \\
\hline Asymptotic Expansion & 8 & 15 & 23 & 62 \\
\hline Continued Fraction & 4 & 5 & 8 & 10 \\
\hline
\end{tabular}

\section{Range}

For the function $e^{x} E_{n}(x)$, the sum of $x$ and $n$ must be less than or equal to the maximum machine value. If the sum is greater, both $e^{x} E_{n}(x)$ and $E_{n}(x)$ are set equal to zero.

For the function $E_{n}(x)$ the range of $x$ is dominant and essentially equivalent to the range for the exponential function. In single precision on the Univac $1108, E_{n}(x)=0$ beyond $x \approx 85$; in double precision beyond $x \approx 704$.

\section{Accuracy}

Using the Univac 1108 in computing $e^{x} E_{n}(x)$, we find the maximum relative error is $1.3(-7)$ for the single precision computation and 2.4. $(-17)$ for the double precision computation.

In computing $E_{n}(x)$, largely due to the error of the exponential routine, the maximum relative error is $4(-7)$ for the single precision computation and $4.5(-16)$ for the double precision computation.

The number of accurate binary digits is essentially the lesser of NBM-NBM ${ }^{1 / 2}$ or $\mathrm{NBM}-\mathrm{I}$ where $\mathrm{I}$ is the number of binary digits representing the integer part of $x$.

\section{Precision}

The precision may be set lower than the maximum by varying NBM or if desirable, deleting NBM and setting the proper value of TOLER. 


\section{Timing (Seconds-Univac 1108)}

\begin{tabular}{l|c|c}
\hline \hline $\begin{array}{l}\text { For } n=1,2,20 \\
\text { over the range }\end{array}$ & $\mathrm{NBM}=27$ & $\mathrm{NBM}=60$ \\
\cline { 2 - 3 } $\begin{array}{l}x(0.02) 1 \text { (153 values) } \\
x=2(1) 85 \text { (252 values) } \\
x=85(10) 715 \text { (192 values) }\end{array}$ & 0.18 & 0.46 \\
Maximum Time/Evaluation & -19 & .61 \\
& & .28 \\
\hline
\end{tabular}

\section{Testing}

The double precision results obtained were compared against available published values. Further check values were obtained by utilizing multi-precision packages. In addition, double precision check values were obtained, where appropriate, with the asymptotic expansion, numerical integration, and the "odd" form of the continued fraction. Single precision results were then checked against the double precision results. In all cases, the results obtained agreed within the reported accuracy.

\section{Driver Program and Its Results}

In the appendix we have included a driver program and its results. The usage of the subroutine is thus illustrated and a reasonable set of check values given to simplify the checkout of modifications to the subroutine. Two tables are given; one for the function $E_{n}(x)$ and the other for $e^{x} E_{n}(x)$. The functions are tabulated to 18 significant figures for $n=1,2$, and 20 , for $x=0,10^{J}\left(10^{J}\right) 10^{J+1}$ with $J=$ JBEGIN(1) JEND. The value of JBEGIN has been set at -2 ; the value of JEND is the minimum value of $J$ for which $E_{1}(x)=0$.

\section{Special Constants}

$E_{1}(1)=0.21938393439552027367716377546012164$

$\sum_{1}^{\infty}(-1)^{n+1 / n} \cdot n !=E_{1}(1)+\gamma=0.79659959929705313428367586554252408$

$$
\begin{gathered}
\Psi(1)=-\gamma(\text { Euler's constant }) \\
=-0.57721566490153286060651209008240243 \\
\Psi(2)=1-\gamma \\
=0.42278433509846713939348790991759756 \\
x=e^{\Psi(1)}=0.56145948356688516982414321479088078 \\
e^{\Psi(2)}=1.52620511159586388047488871503677561 \\
e^{\Psi(3)}=2.51628683093936358025623717559118353 \\
e^{\Psi(4)}=3.51176116633947618136681836314218018 \\
e^{\Psi(5)}=4.50919059491687493725133809381819453 \\
e^{\Psi(6)}=5.50753782970136813780615697003198287 \\
e^{\Psi(7)}=6.50638716436917208390168502548057156 \\
e^{\Psi(8)}=7.50554047605111860659920028057856992 \\
e^{\Psi(9)}=8.50489157986777622381292917641319981 \\
e^{\Psi(10)}=9.50437851808435474744372629150055559 \\
\log _{e} 2=0.69314718055994530941723212145817656 \\
\log _{e} 10=2.30258509299404568401799145468436420
\end{gathered}
$$




$$
\begin{aligned}
2^{-24} & =0.59604644775390625(-7) \\
2^{-27} & =.7450580596923828125(-8) \\
2^{-36} & =.14551915228366851806640625(-10) \\
2^{-48} & =.3552713678800500929355621337890625(-14) \\
2^{-60} & =.86736173798840354720596224069595336(-18) \\
2^{-108} & =.30814879110195773648895647081358837(-32)
\end{aligned}
$$

Maximum and Minimum Machine Values and Their Natural Logarithms

$\mathrm{NBC}=$ Number of binary digits in the (biased) characteristic of a floating point number

$$
\begin{aligned}
& 2^{-\left(2^{\mathrm{NBC}-1}+1\right)} \leqslant x<2^{2^{\mathrm{NBC}-1}-1} \\
& \mathrm{NBC}=8
\end{aligned}
$$

$2^{127}=0.17014 \quad 118346046923173 \quad 168730371588410 \quad(39)$

$2^{-129}=0.14693679385278593849609206715278070 \quad(-38)$

$\log _{e}\left(2^{127}\right)=88.0296919311 \quad 1305429598 \quad 847942518842414$

$\log _{e}\left(2^{-129}\right)=-89.4159862922329449148229436 \quad 6810477728$

$$
\begin{aligned}
& \mathrm{NBC}=11 \\
& 2^{1023}=0.89884 \quad 65674 \quad 31157953864652595394 \quad 51236 \quad(308) \\
& 2^{-1025}=0.27813423231340017288627908966655050 \quad(-308) \\
& \log _{e}\left(2^{1023}\right)=709.0895657128240515338284602 \quad 5171462914 \\
& \log _{e}\left(2^{-1025}\right)=-710.47586007394394215266292449463098227
\end{aligned}
$$

\section{References}

[1] Abramowitz, M. and Stegun, I. A., Handbook of Mathematical Functions, Nat. Bur. Stand. (U.S.), Appl. Math. Ser. 55 , (1964).

[2] Blanch, G., Numerical evaluation of continued fractions, SIAM Review 6, 4, 383-421 (1964).

[3] Clenshaw, C. W., Chebyshev Series for Mathematical Functions, Mathematical Tables, Vol. 5, National Physical Laboratory, 1962.

[4] Cody, W. J. and Thacher, H. C. Jr., Rational Chebyshev approximations for the exponential integral $E_{1}(x)$, Math. Comp. 22, 641-649(1968).

[5] Harris, F. E., Tables of the exponential integral Ei(x), MTAC 11, 9-16(1957).

[6] Lee, K. L., High-Precision Chebyshev Series Approximation to the Exponential Integral, NASA TN D-5953, 1970.

[7] Miller, G. F., Tables of Generalized Exponential Integrals, National Physical Laboratory Mathematical Tables Volume 3 , Her Majesty's Stationery Office, 1960.

[8] Murnaghan, F. D., and Wrench, J. W. Jr., The converging factor for the exponential integral. Report 1535, David Taylor Model Basin, 1963.

[9] Pagurova, V. I., Tables of the Exponential Integral $E_{\nu}(x)=\int_{1}^{\infty} e^{-x u} u^{-v} d u$

(Translated from the Russian by D. G. Fry) (Pergamon Press, New York, 1961).

[10] Placzek, G., The functions $E_{n}(x)=\int_{1}^{\infty} e^{-x u} u^{-n} d u$

Tables of functions and of zeros of functions, Nat. Bur. Stand. (U.S.), Appl. Math. Ser. 37, 57-111 (Nov. 1954).

[11] Wall, H. S., Analytic Theory of Continued Fractions (D. Van Nostrand Co., New York, 1948). 


\section{Appendix}

\section{Implementing Program}

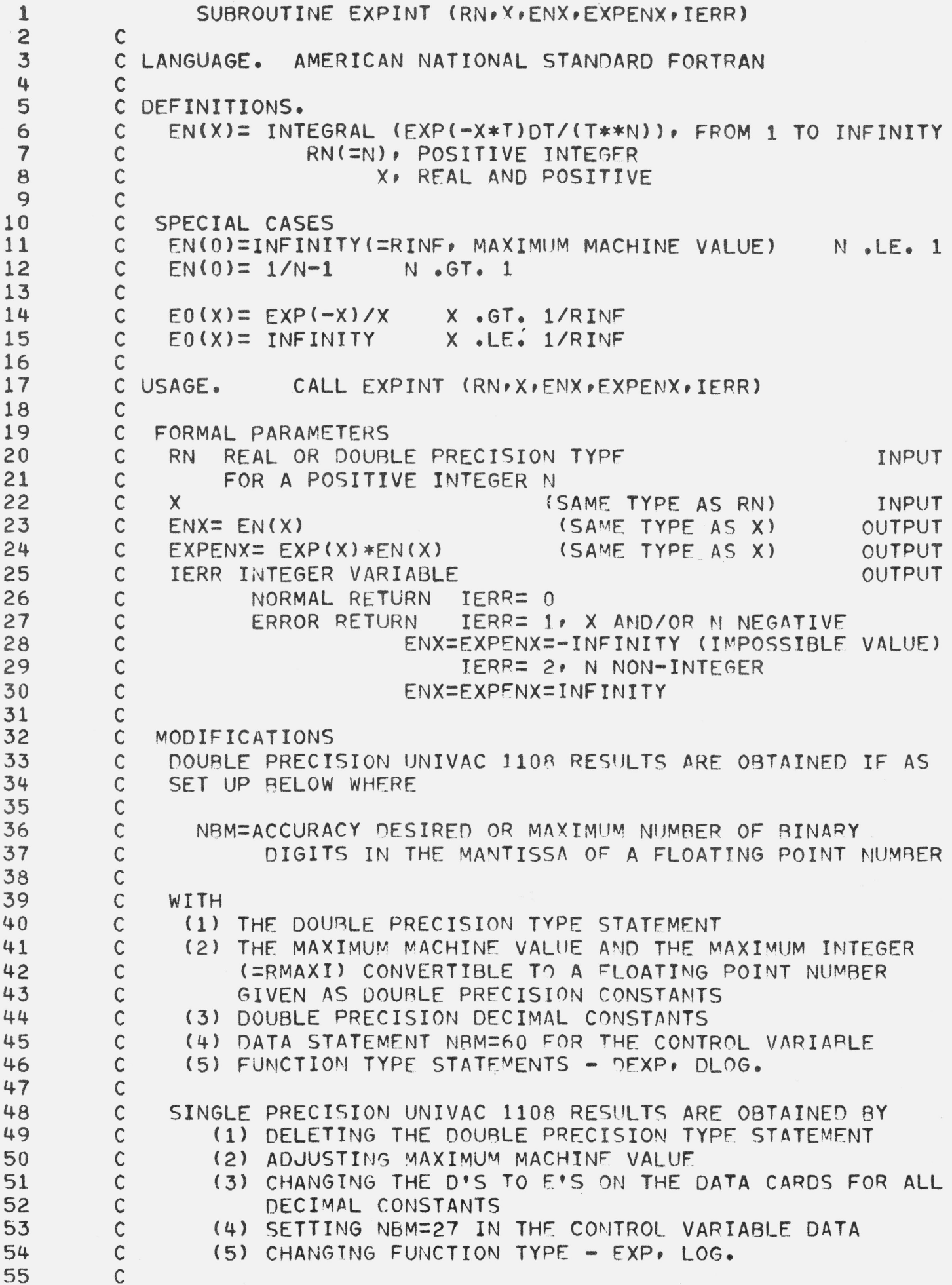




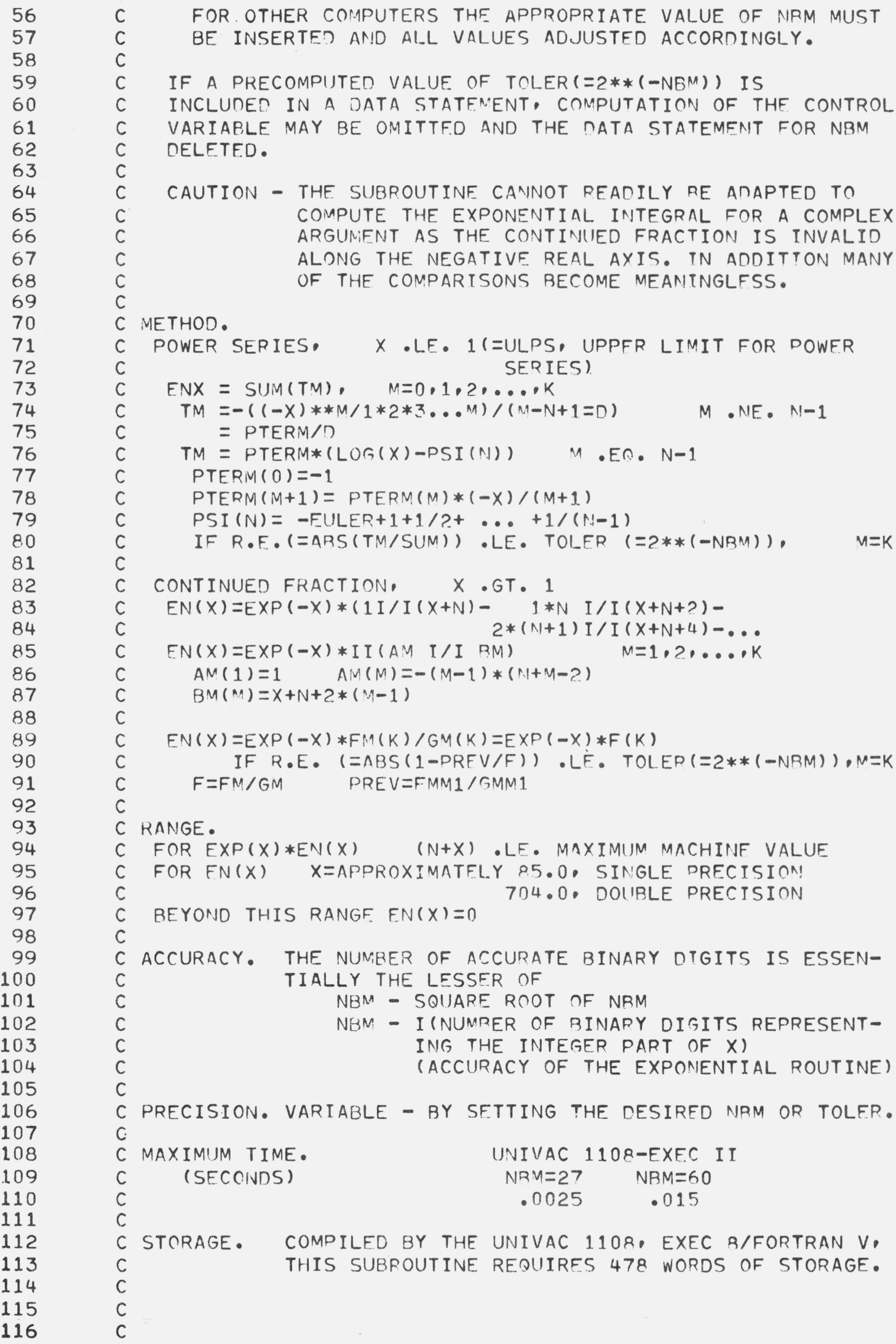




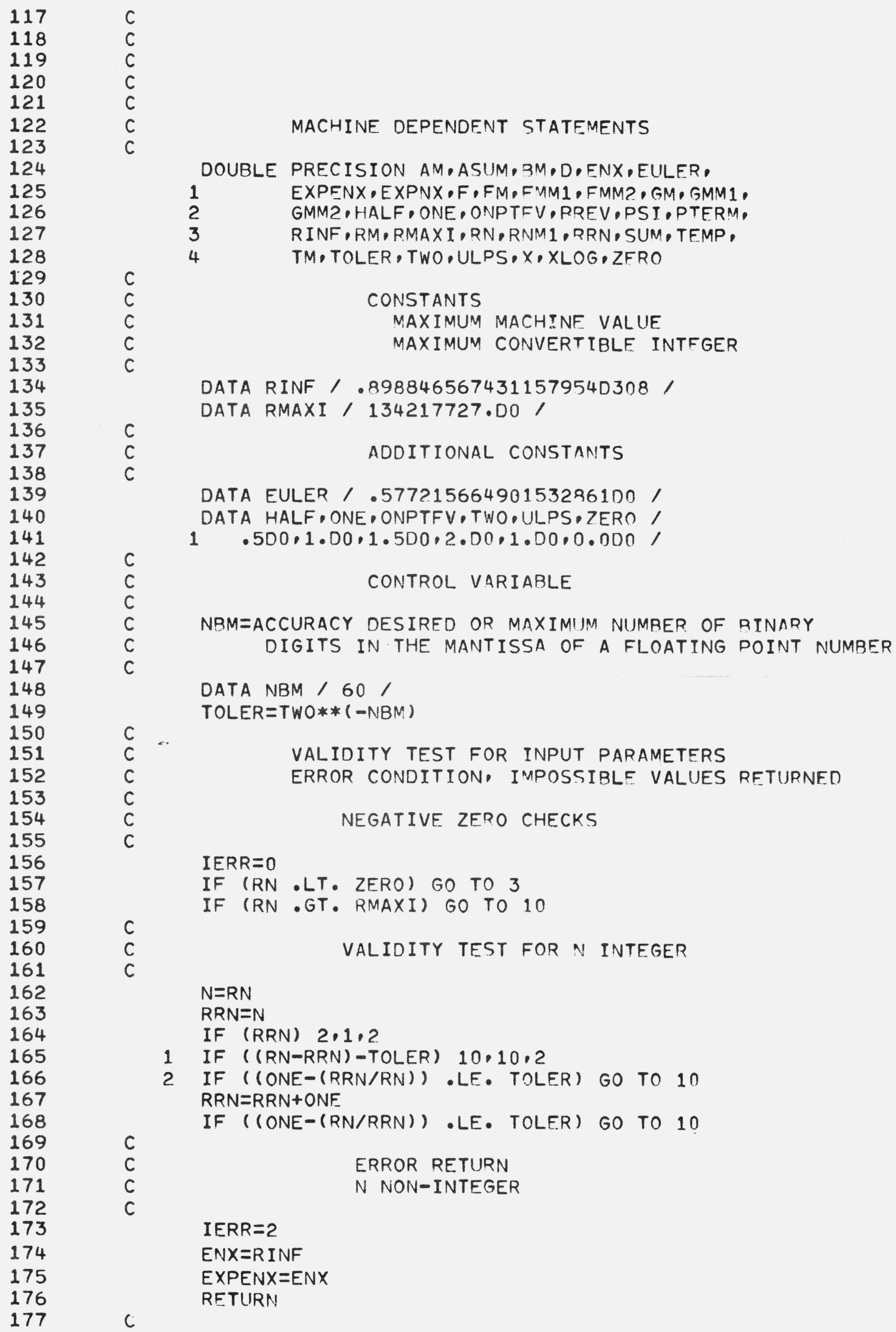




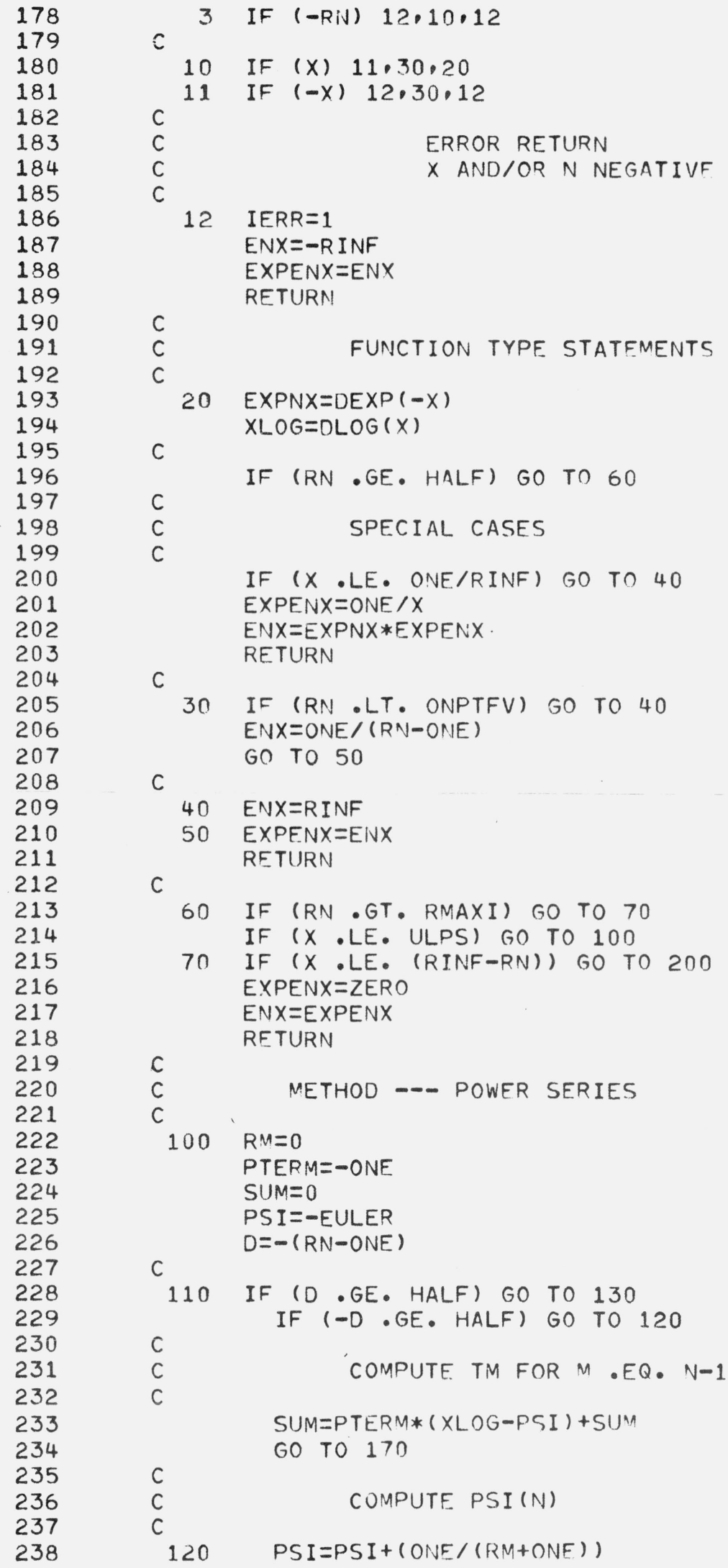




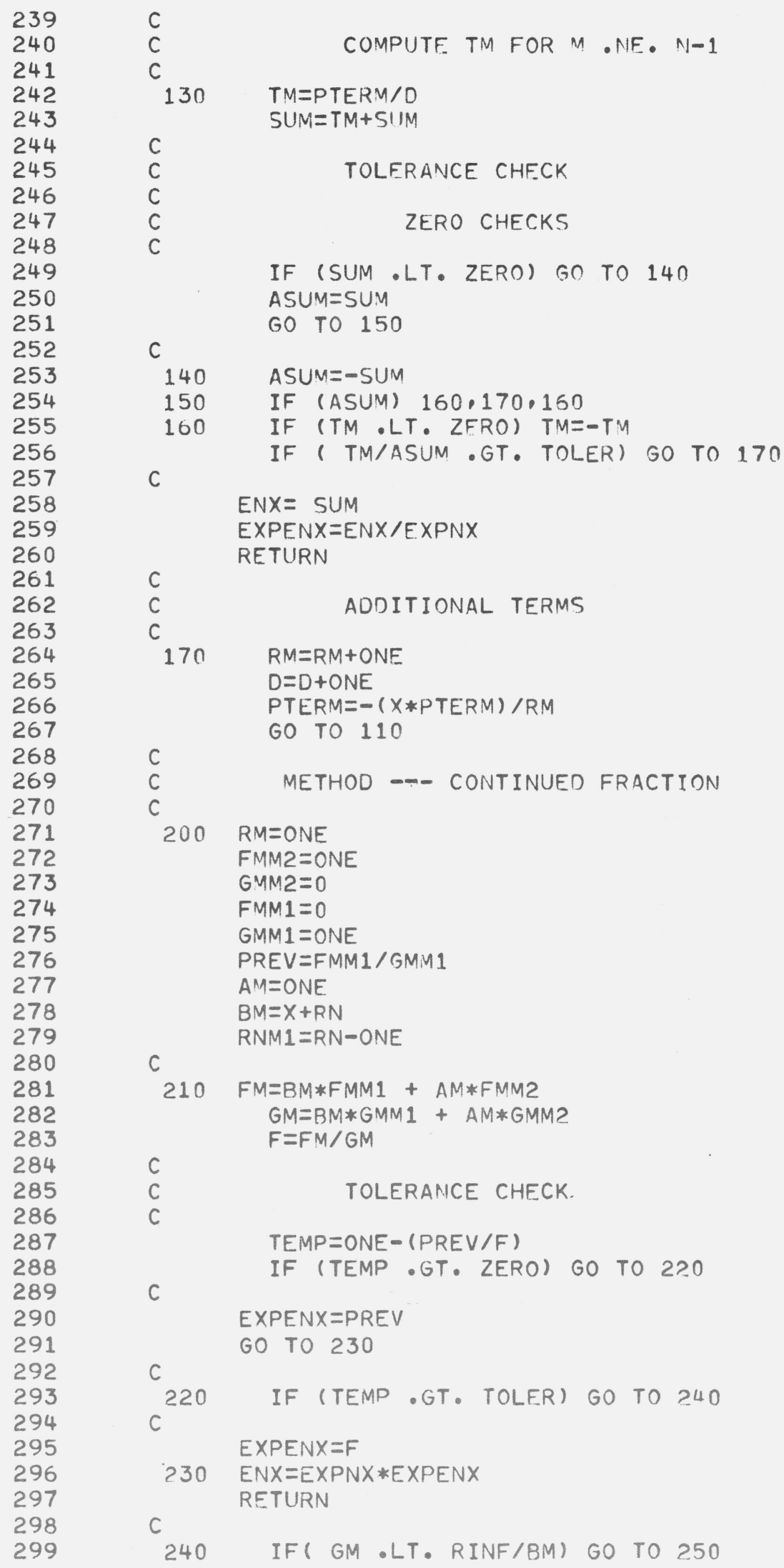


C

$\mathrm{C}$

C BOTH FM AND GM MUST BE TESTED IF N=1 AND $X \quad$. LT. .44

C SCALING SHOULD NOT BE DELETED AS THE VALUES OF FM AND GM

C MAY OVERFLOW FOR SOME CHOICES OF THE PARAMFTERS.

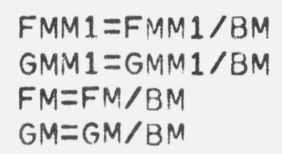




\section{Driver (Test) Program}

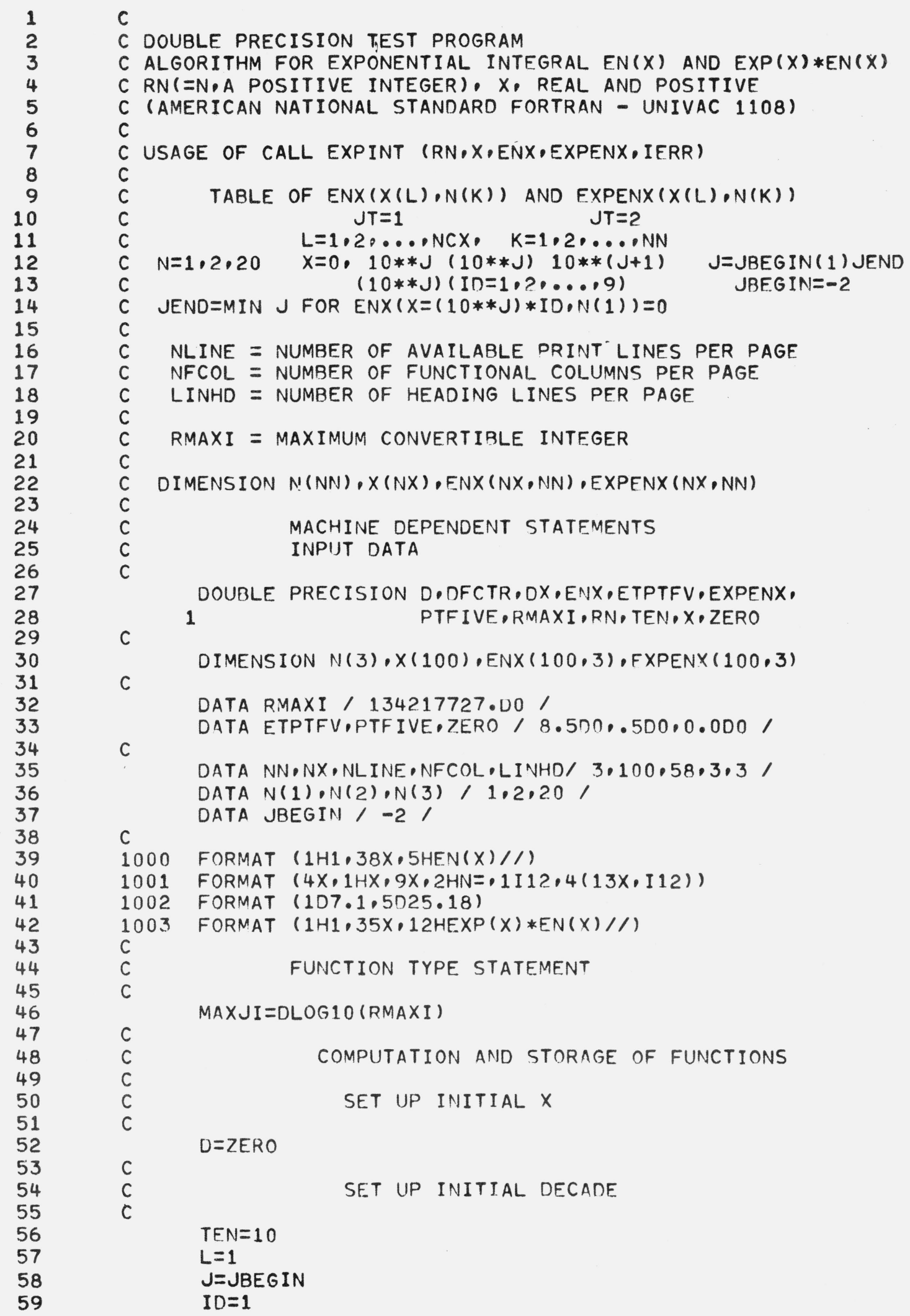




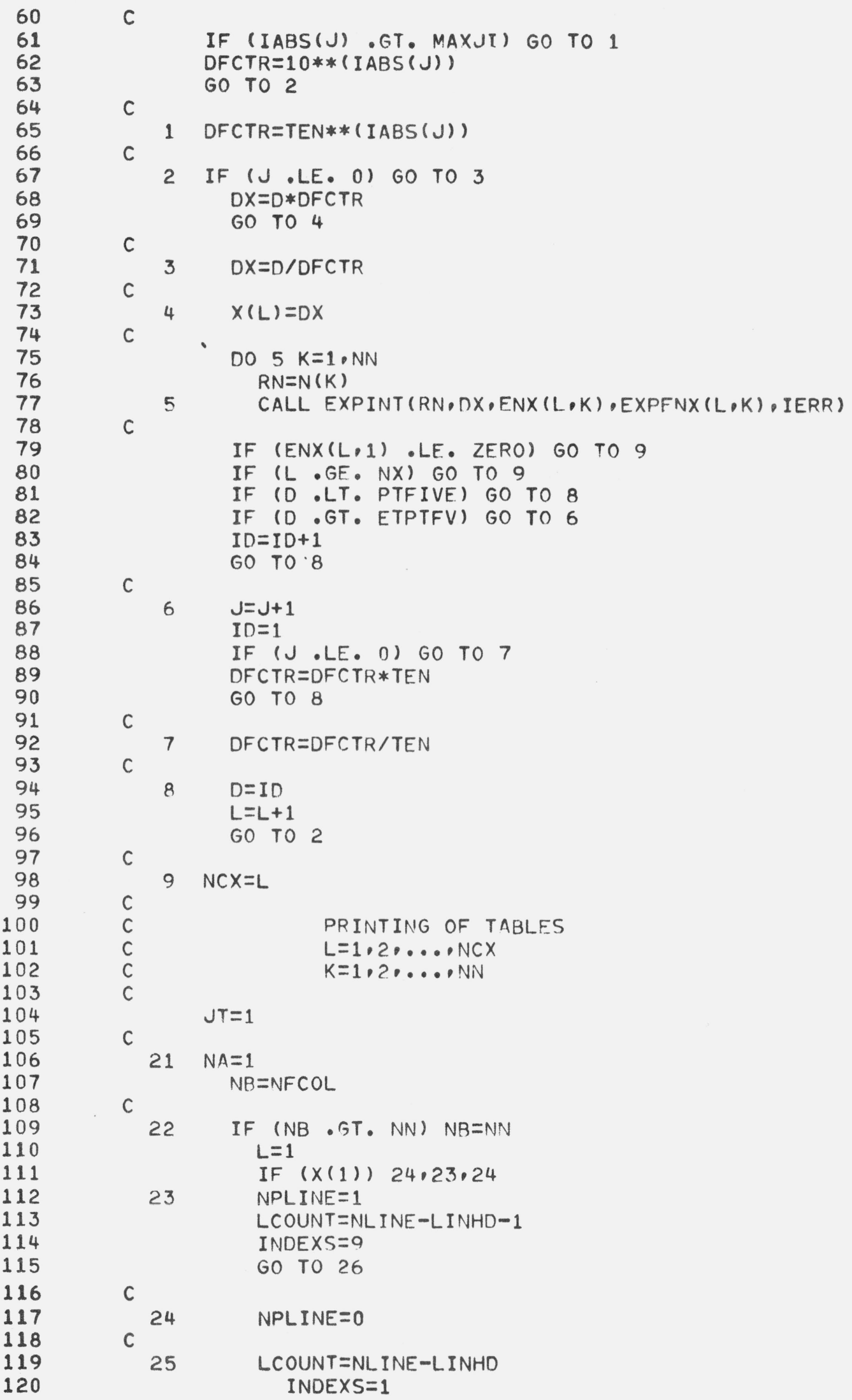




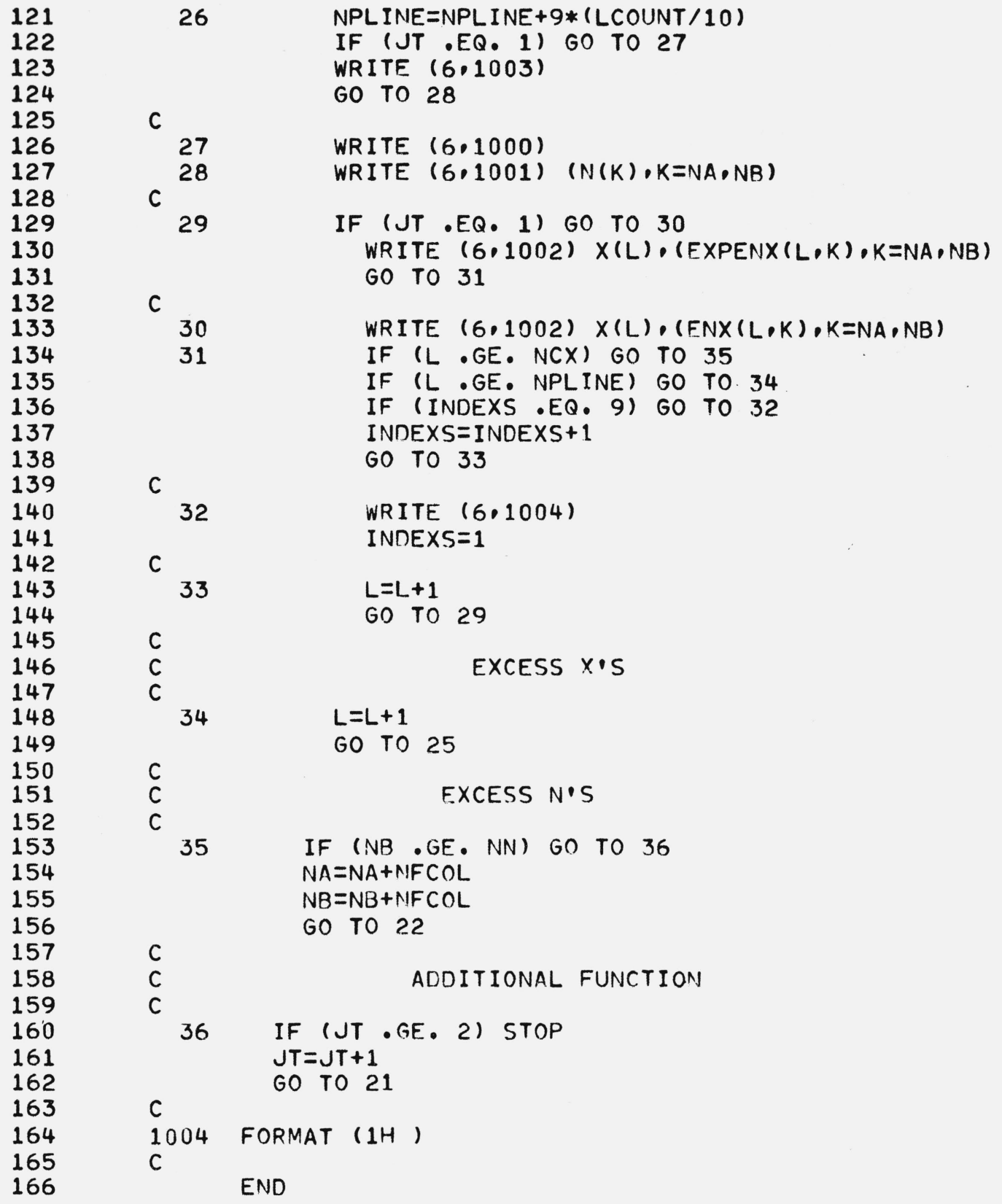




\section{Test Results}

$E N(x)$

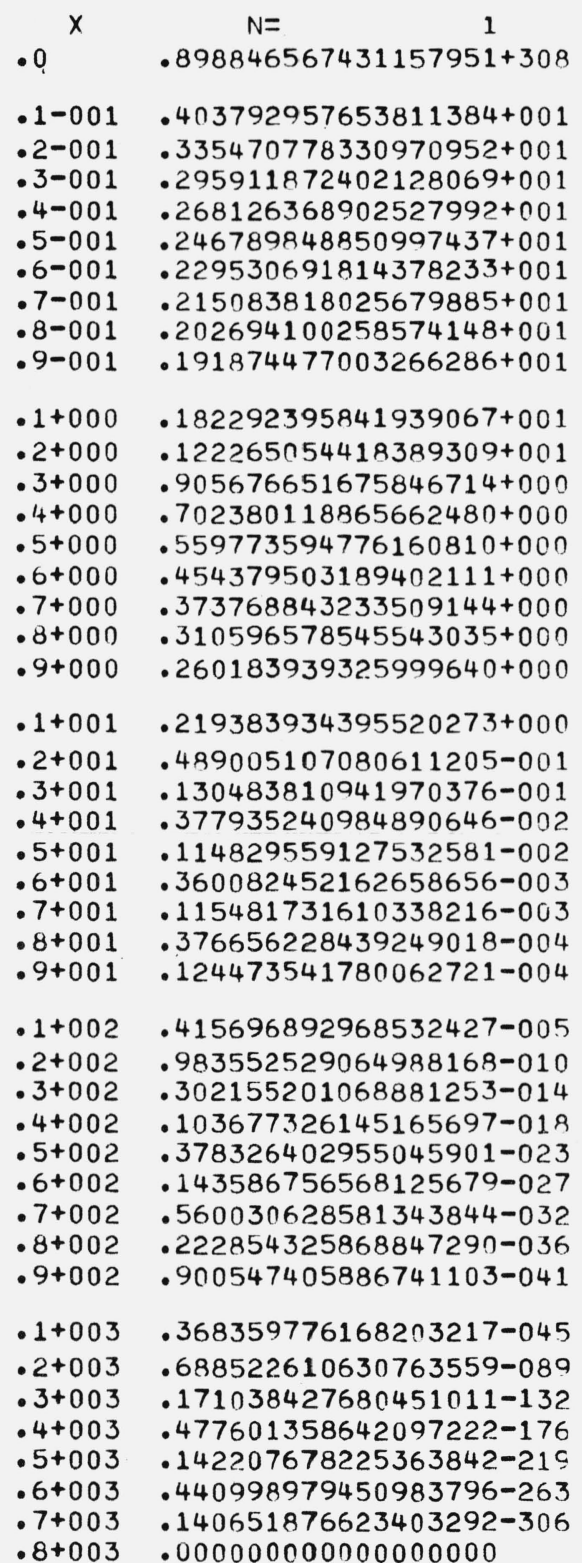

• $898846567431157951+308$

$403792957653811384+001$ $.246789848850997437+001$ $.229530691814378233+001$ . $.182292395841939067+001$ $2065054418389309+00$ $.559773594776160810+000$ $454379503189402111+000$ $.310596578545543035+000$ $.219383934395520273+000$ .489005107080611205-001 $377935240984890646-002$ $114829559127532581-002$ 1 $376656228439249018-004$

415696892968532427-005 .983552529064988168-010 $.378326402955045901-023$ . $143586756568125679-027$ $222854325868847290-036$

(1)

.
- $685130547521041110-089$ - $170473919984934340-132$ $.476416214561680312-176$ $.141924954730934210-219$ $.440267629840803335-263$ $.140451801215403972-306$ .000000000000000000
20 $.526315789473684210-001$ $.520789541793351476-001$ $.515321496513528285-001$ . 509911038545502872-001 . 504557559326025865-001 $.499260456747777298-001$ $.494019135090578269-001$ . 488833004953339252-001 $.483701483186737100-001$ $.478623992826612893-001$

$.473599963028082897-001$ $.426178656910138496-001$ . 383518490868851917-001 .345140002098048623-001 .310612173936309823-001 $.279547522194935671-001$ .251507681997253041-001 $.226449443896666456-001$ .203821193314687553-001

.183459712067558733-001 $.641430585532489937-002$ $.224864806414000919-002$ $.790189099803715192-003$ .278274592885730814-003 . $981884227302490187-004$ $.347068486248843707-004$ . 122877543566663061-004 $.435687424044296172-005$

$.154693627987772485-005$ .521648146507963053-010 . 188625975171560854-014 $.711928232079808894-019$ . $776642308097688871-023$ $.109793192761257778-027$ $.442791440616991968-032$ $.180841147365357070-036$ $.746125498994224778-041$

.310431603951609625-045 .629301793252036997-089 . 160912502553792433-132 $.456044233853820537-176$ $.137021182168391364-219$ $.427505488621821494-263$ .136945221165125589-306 .000000000000000000 


$\begin{array}{ccc}X & N= & 1 \\ .0 & .898846567431157951+308 \\ .1-001 & .407851144345642585+001 \\ .2-001 & .342247737593075321+001 \\ .3-001 & .304923730567447425+001 \\ .4-001 & .279068813598834047+001 \\ .5-001 & .259443034976061332+001 \\ .6-001 & .243724077122346628+001 \\ .7-001 & .230679154487934795+001 \\ .8-001 & .219575897504124847+001 \\ .9-001 & .209944118436360746+001 \\ .1+000 & .201464254470845168+001 \\ .2+000 & .149334874693223961+001 \\ .3+000 & .122253560508058556+001 \\ .4+000 & .104782800845600643+001 \\ .5+000 & .922910632483730466+000 \\ .6+000 & .827933435273508820+000 \\ .7+000 & .752678020029587137+000 \\ .8+000 & .691245397802831489+000 \\ .9+000 & .639949226639299739+000\end{array}$

$.1+001 \quad .596347362323194074+000$ $.2+001.361328616988222592+000$ $.3+001.262083740255318501+000$ $.4+001 \quad .206345649901055832+000$ $.5+001 \quad .170422176284732204+000$ $.6+001.145267629233886893+000$ $.7+001.126641096076632765+000$ $.8+001.112279639253499312+000$ . $9+001.100861955580640929+000$

$.1+002$ $.2+002$ $.3+002$ $.4+002$ $.5+002$ $.6+002$ $.7+002$ $.8+002$ $.9+002$

$.1+003$ $.2+003$ $.3+003$ $.4+003$ $\cdot 5+003$ $.6+003$ $.7+003$ $.8+003$
$.915633339397880818-001$ $.477185454959608417-001$ . 322897387589801252-001 $.244041150796285762-001$ .196151099301148704-001. .163977137080465268-001 . $140872270003268122-001$ . $123475166636786097-001$ . $109903102083356454-001$

$.990194228673301838-002$ .497524632317935662-002 .332229556527070706-002 .249378101793988503-002 .199601590476041089-002 .166389810215794723-002 . 142653641830088669-002 . $124844139167435033-002$
2

$.100000000000000000+001$

$.959214885565435741+000$ $.931550452481384936+000$ . $908522880829765773+000$ $.888372474560466383+000$ $.870278482511969335+000$ $.853765553726592023+000$ - $838524591858445643+000$ . $824339281996700124+000$ $.811050293407275329+000$

$.798535745529154834+000$ . $701330250613552081+000$ $.633239318475824335+000$ $.580868796617597430+000$ $.538544683758134765+000$ $.503239938835894713+000$ $.473125385979289004+000$ $.447003681757734811+000$ $.424045696024630237+000$

$.403652637676805926+000$ . $277342766223554833+000$ . $213748779234044509+000$ $.174617400395776667+000$ . $147889118576338992+000$ . $128394224596678636+000$ . $113512327463570647+000$ . $101762885972005506+000$ . $922423997742316384-001$

.843666606021191810-001 $.456290900807831660-001$ .313078372305962434-001 . 238353968148569492-001 . 192445034942564817-001 .161371775172083932-001 .138941099771231436-001 .121986669057112264-001 .108720812497919106-001

. 980577132669915934-002 $.495073536412867515-002$ .331133041878788067-00? .248759282404598474-002 .199204761979455498-002 .166113870523165916-002 .142450718937931575-002 .124688666051973815-002
20

.526315789473684210-001

. 526023563704061981-001 . 525731681287694728-001 $.525440141582159931-001$ . 525148943946742833-001 $.524858087742430369-001$ .524567572331905115-001 .524277397079539275-001 . 523987561351388700-001 .523698064515186901-001

.523408905940339148-001 .520535787019039881-001 .517695812767576684-001 . 514888379273828789-001 .512112898107201955-001 . 509368795793975367-001 . 506655513315264279-001 $.503972505626390015-001$ $.501319241196527574-001$

.498695201567573519-001 .473956658006950287-001 .451653037195386256-001 $.431428630256384291-001$ $.412996114281546988-001$ $.396120369170190107-001$ .380606810266053526-001 .366292794923112188-001 .353041176322005723-001

.340735390554722559-001 .253085524935690803-001 .201574668908614936-001 . 167577416876913709-001 .143430890423517574-001 .125384637484814277-001 . $111381471287998954-001$ $.100197250911190282-001$ $.910574016947094732-002$

.834476515943799403-002 .454731825027371299-002 .312560692284812426-002 .238122114425943504-002 . 192321864972550092-002 . 161298688732910590-002 . 138894233048444955-002 . $121954838359794140-002$

(Paper 78B4-415) 